\section{Percepciones y factores asociados a la salud bucal y la atención odontológica en el periodo perinatal en las mujeres y sus bebés}

\section{Perceptions and associated factors with the oral health and dental care in perinatal period in women and their babies}

\begin{abstract}
Resumen
Objetivo. Comprender los factores asociados a la salud bucal y la atención odontológica en el periodo perinatal en las mujeres y sus bebés. Métodos. Soportados en la teoría crítica y la salud colectiva, se realizó un estudio con abordaje cualitativo y cuantitativo. El cualitativo, sustentado en el interaccionismo simbólico, se hicieron entrevistas en profundidad sobre18 mujeres en postparto. En el cuantitativo mediante un estudio observacional de corte-transversal sobre 40 mujeres. Las variables explicativas y de salida se relacionaron mediante test de $\mathrm{Chi}^{2}$ para variables cualitativas y prueba t de Student para las cuantitativas. Resultados. En el cualitativo las mujeres dan importancia estética a la salud bucal, sin relacionarla con su salud general y del bebé; la atención odontológica es difícil durante el postparto y no la consideran importante para sus bebés. En el cuantitativo, $15 \%$ de las mujeres no asistieron a la consulta odontológica durante el embarazo, asociándose con el número de días de nacido del bebé. El 82,5\% de las mujeres no asistieron durante el postparto, asociándose con régimen de afiliación en salud. El 72,5\% de las mamás no percibía la necesidad de atención en sus bebés, asociándose a carecer de dinero para el transporte. Conclusiones. Los resultados indican la necesidad de promover mejores construcciones sobre salud bucal y atención odontológica en las mujeres evaluadas. Variables sociodemográficas, posición social, salud bucal de las mujeres se asociaron con inasistencia a la consulta odontológica durante el embarazo, el periodo postparto y la percepción de la madre de la necesidad de tratamiento del bebé
\end{abstract}

Palabras clave: Percepción; Atención odontológica; Embarazo; Periodo postparto; Infante (fuente: DeCS BIREME).

\begin{abstract}
Objective. To understand the factors associated with oral health and dental care in the perinatal period in women and their babies. Methods. Supported in the critical theory and collective health, a qualitative and quantitative study was carried out. In the qualitative one, supported by symbolic interactionism, interviews were conducted on 18
\end{abstract}

\section{Artículo Original}

Sonia Constanza Concha Sánchez ${ }^{1, a}$, Andrea Johanna Almario Barrera ${ }^{1, b}$, Hernando Pabón Ordoñez ${ }^{2, c}$

${ }^{1}$ Universidad Santo Tomás, Grupo de Investigación Salud Integral Bucal, Bucaramanga, Colombia.

2 Universidad Santo Tomás, Bucaramanga, Colombia.

a Doctora en Salud Pública.

${ }^{\text {b } M a g i ́ s t e r ~ e n ~ O d o n t o l o g i ́ a . ~}$

${ }^{c}$ Especialista en Gestión de Empresas.

\section{Correspondencia:}

Sonia Constanza Concha Sánchez: sonia.concha@ ustabuca.edu.co

Carrera 29 \#50-21 Barrio Sotomayor, Bucaramanga,

Colombia

ORCID: 0000-0003-3092-520X

\section{Coautores:}

Andrea Johanna Almario Barrera: andrea.almario@ ustabuca.edu.co

ORCID: 0000-0002-8020-4091

Hernando Pabón Ordoñez: odontohernando@gmail.com

Editor:

Yuri Castro-Rodríguez

Universidad Nacional Mayor de San Marcos, Perú.

Conflicto de interés: no existe ningún conflicto de interés en la realización de este trabajo.

Fuente de financiamiento: el presente artículo se deriva del proyecto financiado por la Universidad Santo Tomás denominado "Determinación Social del Derecho a la Salud Bucal de las mujeres en postparto y de sus hijos en Bucaramanga, Medellín y Santiago de Chile"

Recibido: 06/04/20

Aceptado: 06/06/20

Publicado: 04/08/20 
postpartum women. The quantitative one was an observational cross-sectional study on 40 women. The explanatory and output variables for qualitative study were analyzed using Chi2 test and the quantitative using Student's T-test. Results. In the qualitative approach, women give aesthetic importance to oral health, without relating it to their general health and that of the baby; dental care is difficult in postpartum period and they do not consider it important for their babies. In quantitative terms, $15 \%$ of women did not attend the dental care during pregnancy, associated with the baby's born days number. $82.5 \%$ of the women did not attend it during the postpartum period, being associated it with the health affiliation regime. $72.5 \%$ of moms did not perceive the need of attention for their babies, being associated it with a money lack for transportation. Conclusions. The results indicate the need to promote better constructions on oral health and dental care for the evaluated mothers. Sociodemographic variables, social position, and women's oral health were associated with non-attendance of the dental attention during pregnancy, the postpartum period and the mother perception of the baby's need for treatment.

Keywords: Perception; Dental care; Pregnancy; Postpartum period; Infant (source: MeSH NLM).

\section{Introducción}

La salud bucal es un componente esencial en la vida y la salud de todas las personas, que, adquiere una dimensión especial en la mujer durante el embarazo y el postparto; así como en el bebé. El presente trabajo, analiza en forma particular el tiempo comprendido entre el quinto meses antes del nacimiento y los primeros meses después del parto, que aludirían al periodo perinatal y que representa un momento crucial para la salud general y bucal tanto para la mujer en estas etapas de la vida, como de su hijo.

La literatura científica señala la importancia de la salud bucal y la atención odontológica durante el embarazo por la potencial relación de las infecciones bucales con complicaciones perinatales y por el creciente interés que manifiestan las madres por asumir medidas de cuidado bucal para sí mismas y sus futuros bebés ${ }^{1}$. En el postparto, la evidencia enfatiza en la posible contaminación vertical con microorganismos cariogénicos de madres a hijos, lo que promueve el interés de los profesionales por incentivar los cuidados durante esta etapa de la vida de la mujer y del recién nacido. En la primera infancia, la importancia se soporta en el creciente interés que tienen las madres, por proveer los mejores cuidados bucales a sus pequeños hijos ${ }^{2}$, ya que este es un momento esencial para iniciar prácticas que ayudarán a consolidar los hábitos de autocuidado bucal para toda la vida, aumentando la probabilidad de tener, a futuro, niños y adultos con una mejor salud bucal, y previniendo la caries temprana ${ }^{3}$.

Los programas de promoción de la salud y de prevención de las enfermedades bucales se realizan en diversos ámbitos; sin embargo, la consulta odontológica se constituye en el espacio en que, con mayor frecuencia, se implementan este tipo de actividades, por lo que el acceso de la mujer embarazada, en postparto y del bebé a la consulta odontológica es una estrategia fundamental que debe ser incentivada en el marco del sistema de atención en salud para promover la salud y prevenir las patologías orales ${ }^{4}$.
En Colombia, y en otros países, existen políticas de atención en salud dirigidas a fortalecer el acceso a la atención en salud de la mujer embarazada, en el postparto y en la infancia hasta los cinco años; las políticas que promueven la asistencia a la consulta odontológica de la mujer embarazada son muy claras ${ }^{5}$; sin embargo, el acceso a esta atención durante el postparto y durante los primeros años de vida del niño no lo parecen tanto, lo que dificulta su implementación; adicionalmente, factores individuales, como los conocimientos, las creencias, los temores y el estilo de vida; factores relacionados con el entorno social, como la familia, las redes sociales, el sistema de atención en salud, y los modos de vida condicionan la asistencia y el acceso a la atención odontológica ${ }^{4}$.

En Bucaramanga, una ciudad intermedia ubicada al nororiente de Colombia, hay reportes que señalan la baja frecuencia de asistencia a la consulta odontológica de las mujeres embarazadas ${ }^{6}$; pero, no hay evidencia que señale cómo es esta atención en las mujeres en el periodo postparto, ni en los infantes menores de un año, a pesar de la importancia que representa para la salud, tanto presente como futura de las mujeres y de sus hijos; adicionalmente, no se han identificado los factores que pueden estar influenciando el acceso a la atención bucal en las mujeres en los periodos de interés y en sus hijos en la primera infancia.

En el área odontológica, la superación de la mirada biológica dominante, de la naturalización de la vida social, de la subordinación a la clínica y de su dependencia del modelo médico hegemónico hace necesario la identificación de una "nueva positividad" en la articulación de las dimensiones objetiva y subjetiva en el campo social de la salud bucal. La recuperación de la problemática del sujeto no significa la negación de las estructuras; así como, la definición de un modelo conceptual para la odontología no implica la adopción de un marco teórico de referencia exclusivo y excluyente. La valorización de la dimensión subjetiva (cualitativo) de las prácticas de salud y su integración con lo objetivo (cuantitativo), de las vivencias de los usuarios y trabajadores del sector; así 
como de los factores asociados, proporcionan espacios de comunicación y diálogo con otros saberes y prácticas abriendo nuevas perspectivas de reflexión y acción, promoviendo una mejor comprensión de la situación que enfrentan las mujeres y sus hijos durante el periodo perinatal 7 .

El interaccionismo simbólico (IS) se define como una corriente que se sitúa dentro del paradigma interpretativo (rotulado por algunos como cualitativo), es decir que el sentido de la acción social se elabora desde la perspectiva de los participantes y se construye como una producción de sentido dentro de un universo de simbólicos determinados por la sociedad. Los principios de esta teoría se soportan en: la capacidad de pensamiento de los seres humanos, capacidad que está moldeada por la interacción social, a partir de la cual se aprenden significados y símbolos, con base en ellos se actúa y se interactúa, se elige y se establecen pautas sociales ${ }^{8}$. El IS se constituye en una alternativa que supera la investigación tradicional en el campo odontológico, promueve la comprensión de los fenómenos sociales relacionados con la salud bucal y su atención, que al relacionarse con el método epidemiológico permiten una comprensión integral de los fenómenos observados, desde una epidemiología crítica ${ }^{9}$.

Con estas bases, el objetivo del presente trabajo fue, comprender los factores asociados a la salud bucal y la atención odontológica en el periodo perinatal en las mujeres y sus bebés, residentes en Bucaramanga, Santander (Colombia).

\section{Métodos}

Para dar respuesta al objetivo propuesto se realizó un estudio fundamentado en la teoría crítica, soportados en la salud colectiva ${ }^{10}$, con abordaje cualitativo y cuantitativo. El apartado cualitativo se sustentó en el interaccionismo simbólico. Para ello, previo consentimiento informado, se realizaron entrevistas en profundidad a 18 mujeres embarazadas y en postparto mayores de 18 años, de estratos socioeconómicos bajos, residentes en Bucaramanga y su área metropolitana.

Como categorías de análisis se exploraron sus percepciones sobre la salud bucal, la atención odontológica, en su relación con otras mujeres en embarazo, en postparto, con los odontólogos que las atienden y las instituciones que les prestan la atención.

Las mujeres con cinco o más meses de embarazo y hasta con cuatro meses de postparto, de estratos socioeconómicos bajos se incluyeron en el estudio y se visitaron en sus hogares. Se excluyeron aquellas que registraban alteraciones auditivas, cognitivas o mentales. $\mathrm{Al}$ momento de la visita se obtuvo el consentimiento para vincularse a la investigación, se procedió a efectuar las entrevistas en profundidad a las mujeres de acuerdo con un formato semiestructurado que guiaba el proceso, que fue previamente validado, y cuyas preguntas se fueron profundizando de acuerdo con las respuestas emitidas por las participantes.
La información se recolectó en audio, se transcribió y se analizó en Atlas ti 6.0.15 en forma paralela con su recolección, mediante codificación abierta y categorización basada en el método de comparación constante y muestreo teórico, hasta lograr la saturación de las categorías. Los resultados se validaron en conversatorios con las mujeres.

En el apartado cuantitativo se realizó un estudio observacional analítico de corte transversal con información que se recolectó en una sola visita que se realizó entre julio de 2017 y mayo de 2018. La población la integraron mujeres en postparto y sus bebés, del área metropolitana de Bucaramanga. La muestra incluyó a la totalidad de mujeres en postparto $(n=40)$, cuyos hijos registraban no más de cuatro meses de nacidos, vinculadas a dos instituciones responsables de la implementación de la política "De cero a siempre", por la que se garantiza la protección integral del menor de cuatro años y de la mujer embarazada y postparto en Colombia, que cubre a población vulnerable de estratos socioeconómicos bajos ${ }^{11}$.

Las variables que se recolectaron tanto en las madres como en los niños fueron las siguientes:

Sociodemográficas: edad de la madre recolectada en ańos cumplidos; edad, recolectada en días de nacido, y sexo del bebé. En la madre se exploró también el estrato socioeconómico, la posición social de acuerdo con los parámetros definidos por Arrivillaga et al ${ }^{12}$.

Condición sistémica: en las que se interrogó sobre los antecedentes médicos tanto de la madre como del hijo.

Condición bucal: en las que examinó la presencia de caries evaluada mediante el índice COPD y COPD modificado, la condición periodontal evaluada mediante el índice periodontal comunitario de necesidad de tratamiento (ICPNT), uso y necesidad de prótesis, y la presencia de labio y/o paladar fisurado; esto último se evalúo tanto en la madre como en el bebé, dada su relativa alta frecuencia en la región de estudio; en el bebé también se exploró la presencia de alteraciones dentales y de mucosa oral; todo esto, de acuerdo a lo establecido en el Cuarto Estudio Nacional de Salud Bucal-ENSAB IV-que se implementó para identificar las necesidad en materia en salud bucal en Colombia ${ }^{13}$.

Inserción social, modos y estilo de vida: que incluía criterios relacionados con quién era el responsable económico del hogar, sexo, labor realizada, tipo de contrato y fuente de ingreso del responsable, rango de ingreso del hogar, título de propiedad de bienes, actividad laboral de la mujer entrevistada, estatus laboral de esta, cesación del trabajo por el embarazo o el postparto, jornada laboral de la mujer, disfrute de licencias o permisos de maternidad, recepción de dinero mensual aportado por su pareja, afiliación al sistema de atención en salud, nivel educativo, servicios públicos con los que cuenta, tipo de vivienda, propiedad de la vivienda, nivel de seguridad percibida, número de personas con las que convive, número de habitaciones del hogar, adscripción a alguna organización social, vinculación del niño al sistema de atención en salud, preguntas que se adaptaron del cuestionario de inserción social aplicado en el ENSAB IV ${ }^{13}$. 
Asistencia a la consulta odontológica: Asistencia y percepción de necesidad de atención odontológica durante el embarazo, culminación del tratamiento odontológico. Asistencia a la consulta odontológica en el postparto, solicitud de cita odontológica en postparto, y terminación del tratamiento en el postparto, satisfacción frente a la atención, recepción de educación/ consejería para el cuidado bucal a la madre y/o del hijo. Percepción de necesidad de atención odontológica del bebe en el primer año de vida, solicitud de cita para su hijo, atención del hijo, satisfacción frente a la atención odontológica de su hijo, razones para negar la atención odontológica a su hijo.

Para la recolección de los datos se procedió de la siguiente manera:

Inicialmente, se presentó, concertó y obtuvo el consentimiento para la aplicación de la encuesta y realización del examen bucal de cada madre y su bebé, examen que se realizó en el hogar de las mujeres adscritas a las dos instituciones de Bucaramanga responsables de la implementación de la política "De cero a siempre".

Previo al examen bucal de la madre se entregó el kit de higiene bucal, se procedió a efectuar cepillado supervisado, se verificó la eliminación efectiva de la placa dental. Al momento del examen, la mujer se ubicó en posición acostada, el área de trabajo se iluminó mediante una fuente de luz artificial proveniente de una lámpara ubicada en la frente del examinador. Para el examen de la cavidad bucal, mediante una sonda OMS, el examinador inicia la evaluación en el segundo molar superior derecho, avanza hasta el segundo molar superior izquierdo, continua con el segundo molar inferior izquierdo hasta el segundo molar derecho; las superficies las examina en forma ordenada para asegurar una observación completa, reportando al anotador la peor lesión de caries encontrada para cada estructura. Con la información recolectada se procede a sumar los dientes cariados (con lesiones cavitadas (COP) o no cavitadas (COP modificado)), los obturados y los perdidos que se registran en forma independiente y se procede a sumar estos tres componentes para consolidar el COP y el COP modificado ${ }^{13}$.

Para la evaluación de la condición periodontal el evaluador, mediante una sonda de Wisconsin, explora en los dientes índices (17-14, 13-23, 24-27, 37-34,33-43 y 44-47) el nivel de inserción y profundidad de la bolsa; reportando al anotador el valor más alto para cada sextante; posteriormente, se determinó la condición periodontal, con base a la peor condición evidenciada en alguno de los sextantes ${ }^{13}$.

Para la identificación de la presencia o necesidad de prótesis, el examinador identificó inicialmente la existencia de espacios edéntulos o con restos radiculares, estableciendo la necesidad de prótesis y el tipo de estructura protésica requerida y que refirió al anotador; posteriormente identificó la existencia en boca de prótesis fija, removible, fija y removible, o prótesis total, información que reportó a la persona responsable de su registro. Para terminar el examen bucal el examinador reportó al anotador la presencia o ausencia de labio y/o paladar hendido.

Para el examen bucal del bebe, se procedió inicialmente a entregar a la madre los elementos e indicar la forma de hacer la higiene de la boca de su hijo. Con el bebé acostado apoyando su cabeza en el regazo de la mamá y los pies en las piernas del operador, el examinador procede evaluar y reportar todas las anomalías de mucosa y dentales identificadas en el bebé, incluyendo la presencia de labio y paladar fisurado

Con toda la información recolectada se procedió a sistematizar la información por duplicado en hojas en Excel, se validó la calidad de la digitación mediante la rutina Data Compare del paquete Epi-Info 3.5.4, y se efectuaron los correctivos. La base de datos depurada se exportó al paquete STATA 14.2 para el procesamiento y análisis.

Para el plan de análisis estadístico univariado se calcularon medidas de resumen según la naturaleza de las variables; para las cualitativas se obtuvieron frecuencias absolutas y relativas. Para las variables cuantitativas se calcularon medidas de tendencia central (media y mediana) y de dispersión (rango, varianza y desviación estándar), estas medidas de resumen se presentaron en textos, tablas y gráficos de presentación de resultados. En el plan de análisis bivariado se consideraron como variables de salida la asistencia a la consulta odontológica en el embarazo, el postparto y la necesidad de tratamiento odontológico de sus hijos, que se relacionaron con cada una de las variables explicativas; para ello, se aplicaron test de Chi cuadrado o Exacto de Fisher para las cualitativas y prueba t de Student o test de rangos de Wilcoxon para las cuantitativas, dependiendo de la distribución de los datos, último aspecto que se evalúo mediante la aplicación del test de Shapiro-Wilk. Para todo le análisis se consideró un nivel de significancia de alfa $(\alpha) \leq 0,05$.

La presente investigación se acogió a lo establecido en la resolución 008430 de 1993 que reglamenta la investigación sobre seres humanos en Colombia y a lo establecido en la Declaración de Helsinki, que permitió clasificar esta investigación como una investigación sin riesgo, en la que se respetaron los principios de beneficencia, autonomía, confidencialidad y privacidad de la información. Este trabajo de investigación fue aprobado por el comité de ética de la Universidad Santo Tomás.

\section{Resultados}

\section{Abordaje cualitativo}

A partir del proceso desarrollado, emergieron categorías que dan cuenta de la salud bucal como una construcción social a la que se le concede poca importancia, a la que le otorgan solo valor estético tanto en las mujeres embarazadas como en postparto.

"Cuando estaba embarazada que habia que calzar una muela, me hicieron limpieza... pendiente eran como más de la limpieza...que se vieran blancos..." 
Pocas mujeres establecen relación entre su salud bucal, su salud general y la de su bebé.

"...lo único que a veces siento es una sensibilidad, pero porque la muela... o sea, ella se me huequeó... pero yo estoy bien..."

..si la encía me sangra, pero no creo que eso me enferme ni a mi bebé..."

La atención odontológica representa para las mujeres una situación que para algunas es traumática, frustrante, pero para otras un espacio para su propio cuidado.

“..si es gratis... pero yo no voy (a odontología) porque me da miedo..."

"me colocaron unas calzitas (término empleado por la mujer para referir obturaciones o empastes), me limpiaron y todo porque si me dijo que tenía como calculitos por ahi, me gusto"

Las mujeres tienen, en términos generales, una relación positiva con los profesionales que las atienden; aunque no perciben que el odontólogo les brinde una educación adecuada, que les permita alcanzar una buena salud bucal para ella y su bebé.

"...me limpiaron, me miraron si tenía placa, si pa calzar muelas, pero ya, no más..."

"...Que la odontóloga me diga, "ah tienen un bebé, tiene que tener éste y éste cuidado", no..."

perciben que las instituciones promueven el acceso a la atención odontológica de las mujeres embarazadas, pero no facilitan esta atención durante el postparto que, aunado a sus preocupaciones por su bebe, hacen que a las mujeres con hijos menores de seis meses no les resulte fácil dar importancia a su salud bucal y acceder a la consulta odontológica.

"Solo comencé a ir cuando estaba embarazada a lo de los dientes porque es gratis, porque no le cobraban a uno ..."

"...entonces todo lo que haga la mujer embarazada no le cobran, pero ya no estoy embarazada..."

Con el agravante que las mujeres a pesar de reconocer sus necesidades en materia de salud bucal optan por aplazar sus cuidados permitiendo que los problemas se agraven y se agudicen

“...Si me han invitado, pero no he ido; la próxima es a sacarme las cordales, pero ahora con Sarita lactando... entonces no he ido por eso..."

"...no queria pedir citas diferentes a las que tuvieran que ver con el cuidado de mi bebé..."

\section{Abordaje cuantitativo}

\section{Análisis univariado}

Variables sociodemográficas. Se evaluaron 40 mujeres que se encontraban en los cuatro primeros meses de postparto y a sus hijos. Las mujeres registraban una media de edad de 24,7 $\pm 6,9$ años, predominaron las mujeres entre los 21 y 30 años (50\%). El 40\% decía pertenecer al estrato socioeconómico tres, pero el 92,5\% registraron una posición social baja de acuerdo con lo definido por Arrivillaga et al ${ }^{12}$; en cuanto al nivel educativo, el $37,5 \%$ refirieron haber completado secundaria (Tabla 1), el 57,5\% señalan que están afiliadas al régimen subsidiado (Tabla 2). Los bebes registraron promedio de 114,9 $\pm 57,6$ días de nacidos y el 52,5\% eran niñas (Tabla 1).

Variables relacionadas con la salud bucal, la asistencia a la consulta y percepción de necesidad de atención odontológica del bebé. El 85\% de las mujeres asistieron a la consulta odontológica durante el embarazo, el $82,5 \%$ refieren que no asistieron a esta consulta en el periodo de postparto y el $72,5 \%$ perciben que su hijo menor de un año no necesita de tratamiento odontológico (Tablas 1, 2, 3 y 4 ).

A nivel bucal, se evidenció que las mujeres registraron una media de caries de 1,6 $\pm 1,7$ dientes con caries y de caries modificada de 6,8 $\pm 4,0$. Un promedio de $4,1 \pm$ 3,5 dientes obturados, un COP de 6,2 $\pm 4,2$ y de COP modificado de 11,6 \pm 4,0 dientes cariados, obturados y perdidos. El 95\% de las mujeres no registraban prótesis, el $70 \%$ no requería de esta y el $15 \%$ rehabilitación de una unidad. A nivel periodontal el $72,5 \%$ de las mujeres registraron cálculo dental, por lo que requerían detartraje (Tabla 4). Todos los niños eran edéntulos, ninguna mamá y ni ningún bebe registró labio o paladar fisurado.

\section{Análisis bivariado}

Variables asociadas a la asistencia a la consulta odontológica durante el embarazo. El nivel de escolaridad $(p=0,016)$, la percepción de seguridad del hogar $(p=$ $0,046)$, el sexo del responsable del hogar $(p=0,033)$ y la edad del hijo $(p=0,026)$ se asociaron estadísticamente con la asistencia a la consulta durante el embarazo. Al analizar el nivel de escolaridad se observa que las madres con primaria y secundaria incompleta, y primaria completa no asistieron a consulta odontológica durante la gestación. Las mujeres que consideraban su hogar poco seguro fueron las que, con mayor frecuencia, no asistieron a consulta dental en el embarazo. Todas las mujeres en las que el responsable económico del hogar era una mujer asistieron a esta consulta durante el periodo de gestación. La media y desviación estándar de días de nacido del bebe en las mujeres que durante el embarazo no fueron a la consulta odontológica fue de 67,5 \pm 55,1 (Tabla 1).

Variables asociadas a la asistencia a la consulta odontológica durante el postparto. La vinculación al régimen de seguridad social en salud se asoció en forma estadísticamente significativa con la asistencia a la consulta odontológica durante el periodo del postparto $(p=0,005)$; en este sentido, del total de mujeres que no asistieron a esta consulta en este periodo la mayor proporción estaban adscritas al régimen subsidiado (la atención en salud es cubierta por el estado). También se observó que la media de edad del niño y el promedio de personas con las que conviven fueron menores entre las mujeres que no asistieron a la consulta odontológica, 
Tabla 1. Variables sociodemográficas de las madres, sus hijos y los factores asociados descritos en forma global y según la asistencia a la consulta odontológica de la mujer durante el embarazo

\begin{tabular}{|c|c|c|c|c|c|c|c|}
\hline \multirow{2}{*}{ Variables } & \multirow{2}{*}{ Total } & \multirow{2}{*}{$\%$} & \multicolumn{5}{|c|}{ Asistencia a la consulta odontológica. embarazo } \\
\hline & & & Si & $\%$ & No & $\%$ & $\mathbf{P}$ \\
\hline Total & 40 & 100 & 34 & 85 & 6 & 15 & \\
\hline Edad (años)* & \multicolumn{2}{|c|}{$24,7 \pm 6,9$} & \multicolumn{2}{|c|}{$25 \pm 6,9$} & \multicolumn{2}{|c|}{$22,3 \pm 6,9$} & $0,425+$ \\
\hline \multicolumn{8}{|l|}{ Edad categorizada } \\
\hline$\leq 20$ años & 13 & 32,5 & 11 & 32,4 & 2 & 33,3 & $0,979 \ddagger$ \\
\hline 21-30 años & 20 & 50 & 17 & 50,0 & 3 & 50,0 & \\
\hline 31-50 años & 7 & 17,5 & 6 & 17,6 & 1 & 16,7 & \\
\hline \multicolumn{8}{|l|}{ Estrato socioeconómico } \\
\hline Uno & 14 & 35 & 12 & 35,29 & 2 & 33,3 & $0,786 \ddagger$ \\
\hline Dos & 8 & 20 & 6 & 17,65 & 2 & 33,3 & \\
\hline Tres & 16 & 40 & 14 & 41,18 & 2 & 33,3 & \\
\hline Cuatro & 2 & 5 & 2 & 5,9 & --- & --- & \\
\hline \multicolumn{8}{|l|}{ Posición social (Arrivillaga) } \\
\hline Baja & 37 & 92,5 & 31 & 91,2 & 6 & 100,0 & $0,606 \ddagger$ \\
\hline Media & 3 & 7,5 & 3 & 8,8 & --- & -- & \\
\hline \multicolumn{8}{|l|}{ Nivel escolaridad } \\
\hline Primaria incompleta & 1 & 2,5 & --- & --- & 1 & 16,7 & $0,016 \ddagger$ \\
\hline Primaria completa & 1 & 2,5 & --- & --- & 1 & 16,7 & \\
\hline Secundaria incompleta & 7 & 17,5 & 5 & 14,7 & 2 & 33,3 & \\
\hline Secundaria completa & 15 & 37,5 & 13 & 38,2 & 2 & 33,3 & \\
\hline Técnico/tecnológico & 12 & 30 & 12 & 35,3 & --- & --- & \\
\hline Universitario & 4 & 10 & 4 & 11,8 & --- & --- & \\
\hline \multicolumn{8}{|l|}{ Seguridad del hogar } \\
\hline Seguro & 24 & 60 & 23 & 67,6 & 1 & 16,7 & $0,046 \ddagger$ \\
\hline Poco & 14 & 35 & 9 & 26,5 & 5 & 83,3 & \\
\hline Nada & 2 & 5 & 2 & 5,9 & --- & --- & \\
\hline \multicolumn{8}{|c|}{ Sexo del responsable económico del hogar $(\mathrm{n}=34)$} \\
\hline Femenino & 11 & 32,4 & 11 & 39,3 & --- & --- & $0,033 \ddagger$ \\
\hline Masculino & 22 & 64,7 & 17 & 60,7 & 5 & 83,3 & \\
\hline Ambos & 1 & 2,9 & --- & -- & 1 & 16,7 & \\
\hline \multicolumn{8}{|l|}{ Sexo del hijo } \\
\hline Niña & 21 & 52,5 & 16 & 47,1 & 5 & 83,3 & $0,186 \ddagger$ \\
\hline Niño & 19 & 47,5 & 18 & 52,9 & 1 & 16,7 & \\
\hline Edad del hijo (días)* & \multicolumn{2}{|c|}{$114,9 \pm 57,6$} & \multicolumn{2}{|c|}{$123 \pm 54,6$} & \multicolumn{2}{|c|}{$67,5 \pm 55,1$} & $0,026+$ \\
\hline
\end{tabular}

Tabla 2. Factores asociados a la consulta odontológica de la mujer durante el postparto de Bucaramanga

\begin{tabular}{|c|c|c|c|c|c|c|c|}
\hline \multirow{2}{*}{ Variables } & \multirow{2}{*}{ Total } & \multirow{2}{*}{$\%$} & \multicolumn{5}{|c|}{ Asistencia a la consulta odontológica postparto } \\
\hline & & & $\mathrm{Si}$ & $\%$ & No & $\%$ & $\mathbf{P}$ \\
\hline & 40 & 100 & 7 & 17,5 & 33 & 82,5 & \\
\hline Edad del niño(días)* & \multicolumn{2}{|c|}{$114,9 \pm 57,6$} & \multicolumn{2}{|c|}{$154,3 \pm 43,9$} & \multicolumn{2}{|c|}{$106,5 \pm 57,2$} & $0,052+$ \\
\hline \multicolumn{8}{|l|}{ Régimen de seguridad social } \\
\hline Contributivo & 15 & 37,5 & -- & -- & 15 & 45,5 & $0,005 \ddagger$ \\
\hline Subsidiado & 23 & 57,5 & 5 & 71,4 & 18 & 54,5 & \\
\hline Especial & 1 & 2,5 & 1 & 14,3 & --- & --- & \\
\hline No está afiliado & 1 & 2,5 & 1 & 14,3 & -- & --- & \\
\hline Promedio persona convive* & 2,5 & $\pm 1,8$ & 3,6 & $\pm 1,8$ & 2,4 & $\pm 1,6$ & $0,060+$ \\
\hline
\end{tabular}

*Promedio \pm Desviación estándar 
Tabla 3. Factores asociados a la percepción de las madres de la necesidad de tratamiento en sus hijos menores de un año

\begin{tabular}{|c|c|c|c|c|c|c|c|}
\hline \multirow{2}{*}{ Variables } & \multirow{2}{*}{ Total } & \multirow{2}{*}{$\%$} & \multicolumn{5}{|c|}{ Percepción de necesidad de tratamiento odontológico de menor un año } \\
\hline & & & $\mathrm{Si}$ & $\%$ & No & $\%$ & $\mathbf{P}$ \\
\hline \multicolumn{8}{|c|}{ Ingresos alcanza para cubrir el transporte } \\
\hline Total & 40 & 100 & 11 & 27,5 & 29 & 72,5 & $0,056 \mathbb{I}$ \\
\hline No lo cubre & 28 & 70 & 5 & 45,5 & 23 & 79,3 & \\
\hline Si lo cubre & 12 & 30 & 6 & 54,5 & 6 & 20,7 & \\
\hline \multicolumn{8}{|c|}{ Responsable económico es propietario de: } \\
\hline Total & 33 & 100 & 9 & 17,5 & 24 & 82,5 & $0,038 \ddagger$ \\
\hline Casa o apartamento & 1 & 3,0 & --- & --- & 1 & 4,2 & \\
\hline Otro & 2 & 6,1 & 1 & 11,1 & 1 & 4,2 & \\
\hline No tiene propiedades & 24 & 72,7 & 4 & 44,4 & 20 & 83,3 & \\
\hline No sabe & 6 & 18,2 & 4 & 44,4 & 2 & 8,3 & \\
\hline
\end{tabular}

Test Chi Cuadrado

¥Test Exacto de Fisher

Tabla 4. Condición bucal de las mujeres según la asistencia a odontología durante el embarazo, el postparto y según la percepción de necesidad de atención odontología del bebé

\begin{tabular}{|c|c|c|c|c|c|c|c|}
\hline \multirow[t]{2}{*}{ Variables } & \multirow[t]{2}{*}{ Total } & \multicolumn{2}{|c|}{$\begin{array}{c}\text { Asistencia odontología } \\
\text { embarazo }\end{array}$} & \multicolumn{2}{|c|}{$\begin{array}{c}\text { Asistencia odontología } \\
\text { postparto }\end{array}$} & \multicolumn{2}{|c|}{$\begin{array}{c}\text { Percepción necesidad } \\
\text { odontología hijo menor } \\
\text { un año }\end{array}$} \\
\hline & & No & $\mathbf{P}$ & No & $\mathbf{P}$ & No & $\mathbf{P}$ \\
\hline & $40(100)$ & $6(15)$ & & $33(82,5)$ & & $29(72,5)$ & \\
\hline \multicolumn{8}{|l|}{ Condición dental } \\
\hline Caries* & $1,6 \pm 1,7$ & $1,6 \pm 2,0$ & $0,951+$ & $1,5 \pm 1,8$ & $0,338+$ & $1,9 \pm 1,8$ & $0,048+$ \\
\hline Caries modificada* & $6,8 \pm 4,0$ & $7,3 \pm 3,4$ & $0,741+$ & $6,7 \pm 3,9$ & $0,788+$ & $7,6 \pm 4,3$ & $0,044+$ \\
\hline Obturados* & $4,1 \pm 3,5$ & $4,0 \pm 3,1$ & $0,878+$ & $4,2 \pm 3,7$ & $0,928+$ & $3,9 \pm 3,5$ & $0,483+$ \\
\hline Perdidos* & $0,7 \pm 1,4$ & --- & $0,161+$ & $0,7 \pm 1,5$ & $0,633+$ & $0,7 \pm 1,5$ & $0,884 \dagger$ \\
\hline $\mathrm{COP}^{*}$ & $6,2 \pm 4,2$ & $5,7 \pm 3,7$ & $0,834 \dagger$ & $6,2 \pm 4,5$ & $0,843+$ & $6,4 \pm 4,3$ & $0,476+$ \\
\hline COP modificado* & $11,6 \pm 4,0$ & $11,3 \pm 2,3$ & $0,887+$ & $11,5 \pm 4,2$ & $0,987 \dagger$ & $12,2 \pm 4,1$ & $0,073+$ \\
\hline \multicolumn{8}{|l|}{ Presencia de prótesis } \\
\hline No tiene ${ }^{* *}$ & $38(95)$ & $6(100)$ & $1,0 \ddagger$ & $31(94)$ & $1,0 \ddagger$ & $28(96,6)$ & $0,479 \ddagger$ \\
\hline Fija & $1(2,5)$ & -- & & $1(3)$ & & --- & \\
\hline Removible & $1(2,5)$ & -- & & $1(3)$ & & $1(3,4)$ & \\
\hline \multicolumn{8}{|c|}{ Necesidad de elaborar prótesis } \\
\hline No necesita & $28(70)$ & $5(83,3)$ & $0,741 \ddagger$ & $23(69,7)$ & $0,198 \ddagger$ & $20(69)$ & $0,541 \ddagger$ \\
\hline Unitaria & $6(15)$ & $1(16,7)$ & & $6(18,2)$ & & $4(13,8)$ & \\
\hline Multiunitaria & $4(10)$ & --- & & $2(6,1)$ & & $4(13,8)$ & \\
\hline Uni y Multiunitaria & $2(5)$ & --- & & $2(6,1)$ & & $1(3,4)$ & \\
\hline \multicolumn{8}{|l|}{ Alteración periodontal } \\
\hline Normal & $1(2,5)$ & --- & $0,908 \ddagger$ & --- & $0,198 \ddagger$ & --- & $0,544 \ddagger$ \\
\hline Sangrado & $6(15)$ & $1(16,7)$ & & $4(12,1)$ & & $4(13,8)$ & \\
\hline Cálculo & $29(72,5)$ & $5(83,3)$ & & $25(75,8)$ & & $22(75,9)$ & \\
\hline Bolsas 4-5 mm & $3(7,5)$ & -- & & $3(9,1)$ & & $2(6,9)$ & \\
\hline Bolsas 6 mm o más & $1(2,5)$ & -- & & $1(3,0)$ & & $1(3,4)$ & \\
\hline \multicolumn{8}{|c|}{ Necesidad de tratamiento periodonal } \\
\hline No necesita & $1(2,5)$ & --- & $0,799 \ddagger$ & --- & $0,441 \ddagger$ & --- & $0,441 \ddagger$ \\
\hline Educación & $6(15)$ & $1(16,7)$ & & $4(13,8)$ & & $4(13,8)$ & \\
\hline Detartraje & $29(72,5)$ & $5(83,3)$ & & $22(75,9)$ & & $22(75,9)$ & \\
\hline Más complejo & $4(10)$ & --- & & $3(10,3)$ & & $3(10,3)$ & \\
\hline
\end{tabular}


aunque para estas variables no se evidencian asociaciones estadísticas (Tabla 2).

Variables asociadas a la percepción de la necesidad de tratamiento odontológico en el primer ańo de vida. La variable tenencia de propiedad se asoció significativamente con la percepción que tienen las madres de la necesidad de tratamiento odontológico de sus hijos en el primer año $(p=0,038)$ (Tabla 3); también se asociaron con esta percepción la media de caries $(p=0,048)$ y de caries modificada que registraban las madres $(p=0,044)$ (Tabla 4). Para tenencia de propiedad se encontró que del total de madres que no percibieron la necesidad de atención odontológica para sus hijos el 83,3\% no tenían ninguna propiedad. Al referir la condición de caries se observó una media más alta de caries y caries modificada en las mujeres que percibieron que sus hijos no requerían consulta odontológica en su primer ańo de vida, con valores de $1,9 \pm 1,8$ y 7,6 \pm 4,3 dientes cariados respectivamente (Tabla 4 ).

\section{Discusión}

El objetivo de esta investigación fue comprender los factores asociados a la salud bucal y la atención odontológica en el periodo perinatal en las mujeres y sus hijos menores de un año, promoviendo esta comprensión desde una mirada tanto interpretativa como de análisis de la magnitud del fenómeno.

Con base en los resultados derivados del abordaje cualitativo se percibe relación con el trabajo desarrollado por Barón et $a^{14}$, quienes señalan que el proceso salud salud-enfermedad bucal no se asocia a la muerte, pero la enfermedad bucal si se relaciona con dolor, deterioro en la calidad de vida y la solución es la pérdida del diente. Refieren, además, que la belleza de la boca se relaciona con estereotipos en el que los medios de comunicación son un factor que enfatizan en la superficialidad, consumismo y materialismo. Las representaciones colectivas indican la poca importancia que se le otorga a la salud oral por no relacionarse con el resto del cuerpo. La pérdida dental es considerada normal y el odontólogo es un lujo más que una necesidad.

Rengifo, en un trabajo desarrollado sobre mujeres embarazadas, señaló que además del estrato socioeconómico, la dificultad de acceso a los servicios asistenciales, la baja escolaridad y los hábitos de cuidado; las creencias, también pueden llegar a ser muy representativas e influyentes sobre la condición oral de las gestantes, pues condicionan las percepciones de susceptibilidad, severidad, beneficios alcanzados y los comportamientos que se asumen; refirió además, que toda creencia se desarrolla, transmite y mantiene a través de la experiencia del grupo social al que está vinculada la mujer embarazada, influenciadas por el contexto de la familia (abuela-madre-gestante) y el entorno social (gestante-gestante), al que mujer pertenece; la información es comprendida y aprehendida, por las mujeres, de acuerdo a sus características personales y de su entorno social ${ }^{15}$. Por su parte, Escobar et $a^{16}{ }^{16}$, refieren que la gestación y la maternidad son acontecimientos trascendentales en la vida de las mujeres, procesos que desencadenan diversos sentimientos en las futuras madres, a menudo contradictorios, por lo que la salud bucal no parece ser una preocupación central durante el embarazo ${ }^{16}$; en este mismo sentido, Miraschi et al ${ }^{17}$ muestran cómo, a pesar de los problemas de salud oral evidenciados en las gestantes involucradas en su investigación, estos no parecen afectar la calidad de vida en estas mujeres ${ }^{17}$. Coincidiendo con estas apreciaciones, Saddki et al ${ }^{18}$, aseveran que las madres le dan poco valor a la condición y sus cuidados orales, por lo que no perciben la necesidad de asistir consulta odontológica ${ }^{18}$. Resultados que coinciden con los obtenidos en el apartado cualitativo en el presente trabajo.

A partir del abordaje cuantitativo se evidenció que fue baja la proporción de mujeres que dejaron de asistir a la consulta odontológica durante el embarazo; un bajo promedio de días de nacido del bebe, que una mujer fuera la responsable económica del hogar, un bajo nivel de escolaridad de las madres, y la percepción de inseguridad del hogar fueron las variables que se asociaron estadísticamente con la inasistencia a la consulta odontológica en este periodo. Una elevada proporción de mujeres no acudieron a la atención de sus necesidades bucales durante el postparto; estar vinculada al régimen subsidiada fue la variable que se asoció estadísticamente con esta baja asistencia. Por otra parte, el 72,5\% de las madres consideraban que sus hijos no requerían atención dental en el primer año de vida; las variables asociadas fueron el promedio de lesiones cariosas en la madre y el carecer de dinero para el transporte.

En este trabajo se evidenció una elevada frecuencia de asistencia a la consulta odontológica durante el embarazo $(85 \%)$, resultados que parecen contrastar con lo referido por Concha Sánchez y Morales en 2014, pues en su trabajo referían que para la información recopilada en los Registros Individuales de Prestación de Servicios (RIPS) de 2010 el 32\% de las mujeres embarazadas en Santander y el 37\% de las mujeres en Bucaramanga, recibieron atención odontológica ${ }^{6}$. Esta diferencia se podría deber al hecho que cinco años después, el sistema de atención en salud en Santander y específicamente en Bucaramanga logró optimizar el acceso a la atención odontológica en las mujeres durante la gestación. Pero también podría sustentarse en el hecho que las mujeres involucradas en el presente trabajo estaban adscritas a un programa que se soporta en la política de "Cero a Siempre" que busca garantizar la salud en el niño menor de cinco años y de la mujer durante el embarazo, lo que pudo incentivar la atención odontológica de las mujeres gestantes, ya que este tipo de programas obligan a las participantes a vincularse a los paquetes de atención, incluyendo la atención odontológica ${ }^{13}$.

Uno de los factores asociados a la inasistencia a la consulta odontológica durante el embarazo fue la media de edad de la madre y de los bebes; esto podría estar señalando una potencial relación con la experiencia de las madres en el cuidado de sus hijos y en las dificultades que representa para una mujer joven asumir el cuidado de un bebe muy 
pequeño. Las mamás con mayor edad y con niños con más días de nacidos fueron las que accedieron a la consulta odontológica. Amaya et al ${ }^{19}$, exploraron el efecto de la edad de la mujer sobre las potenciales complicaciones durante el embarazo, señalando diferencias asociadas a la edad de las mujeres durante la gestación, su experiencia y madurez para asumir sus cuidados; aspecto que podría estar sucediendo en el presente trabajo, ya que la media de edad de las mujeres involucradas en el presente trabajo y que asistieron a la consulta fue de $25 \pm 6,9$ años y sus hijos registraron un promedio de $123 \pm 54,6$ días, mientras que las madres que dejaron de asistir fue de $22,3 \pm 6,9$ años y sus hijos de $67,5 \pm 55,1$ días ${ }^{19}$. Adicionalmente, Corchuelo-Ojeda y González Pérez en 2014 encontraron que las madres de menor edad asistieron menos a la consulta odontológica que las de mayor edad ${ }^{20}$.

El nivel de escolaridad también se identificó como un factor asociado a la asistencia a la consulta odontológica durante el embarazo y que coincide con lo referido por Corchuelo-Ojeda y González Pérez, cuando señala que una escolaridad completa aumenta 1,7 (IC95\%: $1,1-2,7)$ veces la probabilidad de asistir a la consulta odontológica al compararse con las que registraban secundaria incompleta ${ }^{20}$. Méndez y Pérez ${ }^{21}$, por otra parte, refieren que la demanda en la atención odontológica por parte de las mujeres embarazadas fue baja y no se relacionó con el nivel de conocimientos sobre salud bucal, las campañas educativas fueron insuficientes para promover los conocimientos en las gestantes ${ }^{21}$. Concha Sánchez en un trabajo desarrollado en tres localidades de Bogotá señala que dentro de los procesos críticos del orden cultural en la dimensión particular el acceso y permanencia de las mujeres en el sistema educativo, así como, el machismo, el racismo, la marginación y segregación social son aspectos que condicionan la asistencia a la consulta odontológica de las mujeres durante el embarazo ${ }^{22}$.

Además, en el presente trabajo, la percepción de inseguridad del hogar de las mujeres se asoció con la inasistencia a la consulta odontológica durante el embarazo; aspecto que coincide con los referido por Concha Sánchez en la investigación desarrollada en Bogotá cuando señala que:

"Cuando se analizan los vecindarios como espacios
de reproducción social, es importante reconocer que
el nivel de inseguridad, de violencia, de comercializa-
ción de drogas psicoactivas, aumenta el nivel de vul-
nerabilidad social y determina el tipo de relaciones
que se establecen entre los individuos y los colectivos;
con esta investigación se confirma que los barrios en
los que residen las mujeres embarazadas entrevista-
das no les ofrecen las condiciones de seguridad y de
bienestar que les permitan moverse en los vecindarios
con tranquilidad, ni desplazarse a los centros de salud
para recibir la atención odontológica" 22

Por su parte, Lamarca et al ${ }^{23}$ en 2014 analizaron la relación de la calidad de vida con el capital social en mujeres embarazadas y en postparto, el cuestionario que aplicaron para analizar el capital social incluyó cuatro dimensiones confianza social, control social, seguridad del vecindario y eficacia política, encontrando que las mujeres que residían en vecindarios con alto capital social reportaban mejor calidad de vida relacionada con la salud bucal que aquellas que residían en vecindarios de bajo capital social, lo que podría estar incidiendo en la frecuencia en la asistencia a la consulta odontológica de las mujeres durante el embarazo ${ }^{23}$.

Rocha et $a l^{4}$ en su revisión sistemática, señalan que los ingresos familiares, el nivel educativo y el tipo de aseguramiento en salud condicionan el acceso a la atención odontológica de las mujeres durante el embarazo ${ }^{4}$, aspecto que también es referido por Amin y Elsalhyv ${ }^{1}$ y podría estar influenciando también este acceso en las mujeres en el postparto, pues en la presente investigación, la vinculación a la atención en salud se asoció estadísticamente con la asistencia, de las mujeres en postparto, a la consulta odontológica ${ }^{1}$.

La media de personas con las que conviven las mujeres en postparto que asistieron a la consulta odontológica en Bucaramanga fue mayor $(3,6 \pm 1,8)$ que en las mujeres que no asistieron a esta $(2,4 \pm 1,6)$, aspecto que parece coincidir con lo referido por Gwengu en Sudáfrica, pues en su estudio reportan que la media de personas con las que conviven las mujeres que asistieron a la consulta fue de $5 \pm 2,5$ en tanto que las que no asistieron fue de $3 \pm 2,6$ en los dos estudios no se evidencian diferencias estadísticamente significativas, pero en términos sociales podrían estar indicando, probablemente, que las mujeres en postparto que asistieron a la consulta podrían tener mayor soporte social que aquellas que no lo hicieron; lo que en términos prácticos podría significar que tienen con quien dejar a su hijos menores de un año cuando asisten a consulta dental ${ }^{24}$.

Rodríguez-Páez ${ }^{24}$ et al y Gwengu ${ }^{25}$ al analizar los factores económicos, en particular los recursos que les permitiera el pago de medios de transporte a las maternas y a las mujeres en postparto, se constituyó en una barrera de acceso al Control Prenatal y al acceso a la atención odontológica. Aspecto que, aunque sin ser estadísticamente significativa, emergió como un aspecto relevante y que incidió en la percepción que tienen las madres sobre la necesidad de la asistencia a la consulta odontológica en el menor de un año.

Rowan-Legg ${ }^{3}$. refiere que la mayor carga de la enfermedad bucal se concentra en los niños con mayores desventajas sociales, en particular las familias con bajos ingresos familiares, indicando con ello las inequidades que enfrentan los niños y su influencia el acceso a la atención odontológica pues esta compite con necesidades básicas que podrían hacer pensar a las madres, que la visita al odontólogo podría catalogarse como un lujo ${ }^{3}$.

Franco $e t a l^{26}$, por su parte señalan, en un estudio que exploraba las barreras de acceso a la atención odontológica de los niños, que cerca del $20 \%$ de las madres consideran que sus hijos pequeños registraban regular o mala salud bucal, sin embargo, solo la mitad de ellas pensaron que este era un problema que justificará solicitar la atención 
odontológica para los menores; aspecto que podría coincidir con lo registrado en el presente trabajo ${ }^{26}$. En esta investigación, en la que se exploraba la percepción de las madres de Bucaramanga, se podrían pensar que las participantes podrían estar subestimando la importancia, que el menor de un año asista a la consulta odontológica o que a estas madres no se les brindaron los fundamentos que les permitan reconocer el valor de este tipo de atención en el bebé.

Cuando se analiza la condición dental, en el presente trabajo las mujeres evaluadas registraron un promedio en el COP de 6,2 y de COP modificado de 11,6; mientras que, en el Cuarto Estudio Nacional de salud bucal, en Colombia la media de COP fue de 6,6 y de COP modificado de 14,5 lo que parece indicar que el comportamiento de caries para las mujeres en Bucaramanga y su área metropolitana es similar al referido para las mujeres embarazadas en Colombia ${ }^{13}$.

El 30\% de las mujeres en Bucaramanga, en este trabajo, registran edentulismo y requieren de algún tipo de rehabilitación protésica, mientras que en Colombia el $52,7 \%$ de las gestantes registraron edentulismo, lo que indica que este evento fue menor en las mujeres involucradas en este trabajo con respecto a lo que se evidencia en el país ${ }^{13}$.

En cuanto a la condición periodontal Yllesca-Yllesca y et al. ${ }^{27}$, en Perú señalan que el $100 \%$ de las mujeres que evaluaron registraron sangrado al sondaje y el 33\% bolsas periodontales ${ }^{27}$. Méndez y Pérez ${ }^{21}$ señalan, en mujeres embarazadas en Paraguay, que el 85,4\% registraban gingivitis, predominando la moderada en el $49 \%$ de estas mujeres ${ }^{21}$. En el presente trabajo el $97,5 \%$ registraron alteraciones a nivel periodontal y el $10 \%$ bolsas periodontales; lo que parece indicar que la afección periodontal fue más severa en las mujeres peruanas, pero un mayor compromiso periodontal al registrado por las mujeres en Paraguay. Cartes-Velásquez et al. ${ }^{28}$ en Chile por su parte señalan que el $72,9 \%$ de las mujeres registraban cálculo mientras que en este trabajo la presencia de cálculo se observó en el 72,5\% de las mujeres, lo que parece señalar un comportamiento similar tanto en las mujeres chilenas como en las de Bucaramanga ${ }^{28}$.

En todos los casos se evidencia que la condición bucal de las mujeres embarazadas y en postparto está comprometida y requiere de una mayor atención por parte del odontólogo y del sistema de salud. Adicionalmente, se observa la necesidad de sensibilizar a las madres sobre la importancia de la visita del niño a la consulta odontológica en el primer año de vida.

Es importante señalar que, en el apartado cuantitativo, el número de mujeres incluidas en este trabajo, así como la recolección de la información en un solo momento en el tiempo no permite hacer inferencias más allá de la potencial asociación la asistencia a la consulta odontológica, por lo que se requiere de una muestra que logre representar este fenómeno en las mujeres de Bucaramanga y de su seguimiento constante como una estrategia que permita comprender los eventos de interés a nivel poblacional.
Como fortaleza de la presente investigación se destaca el abordaje cualitativo y cuantitativo que permite comprender además de la magnitud del fenómeno, los imaginarios que tienen las mujeres evaluadas con respecto a su salud bucal y de la asistencia a la consulta odontológica tanto en ellas, como en sus hijos menores de un año.

A partir de los resultados, del abordaje cualitativo, se reconoce la necesidad de crear espacios que promuevan la salud bucal y la atención odontológica de las mujeres durante el periodo del postparto; en el bebé desde que cumple los seis meses de edad, y extenderse hasta los dos o tres años de vida, periodo en el que usualmente ocurre la erupción de la dentición temporal; estas acciones deben conectarse a la formulación de políticas efectivas que incentiven el cuidado bucal de las mujeres en el posparto, de sus bebés y la capacidad de empoderar a estas mujeres para acceder a la atención odontológica; política de la que carece el país en este momento y que señalan la necesidad de promover procesos que superen las estrategias de educación en salud oral, dirigidas a madre e hijos, que se desarrollan en una sola sesión odontológica, que impiden la apropiación por parte de la madre y la replicación en su entorno familiar y social.

Con base en los resultados derivados, del abordaje cuantitativo, desarrollado en el presente trabajo, se puede concluir que las variables sociodemográficas de la madre, las relacionadas con la posición social, la salud bucal de las mujeres se asocian con la inasistencia a la consulta odontológica durante el embarazo, el periodo postparto y sobre la percepción de la madre de la necesidad de atención odontológica en el menor de un año.

A partir de estos resultados se evidencia la necesidad de fortalecer los procesos del orden colectivo y del individual orientados a promover la asistencia de la consulta odontológica en las mujeres en postparto y en el bebé.

\section{Referencias bibliográficas}

1. Amin M, ElSalhy M: Factors affecting utilization of dental services during pregnancy. J Periodontol 2014; 85:1712-1721

2. Mitchell SC, Ruby JD, Moser S, Momeni S, Smith A, Osgood R, Litaker M, Childers N, Volker JF. Maternal Transmission of Mutans Streptococci in Severe-Early Childhood Caries. Pediatr Dent. 2009;31(3):193-20

3. Rowan-Legg A. Oral health care for children-a call for action. Paediatric Child Health. 2013;18(1):37-43

4. Rocha JS, Arima LY, Werneck RI, Moyses SJ, Baldani MH. Determinants of Dental Care Attendance during Pregnancy: A systematic Review. Caries Res. 2018;52:139-152

5. Almario-Barrera AJ, Villarreal-Neira C, Concha Sánchez SC. Aspectos relacionados con el número de citas odontológicas a las que asisten las mujeres embarazadas en Santander. Rev. Fac. Med. 2017;65(1):73-9

6. Concha Sánchez SC, Morales Borrero C. La inequidad en el acceso a la atención odontológica de las mujeres gestantes en Santander. Rev.univ.ind.santander.salud. 2014;46(1):35-45. 
7. Almeida-Filho N, Silva Paim J, La crisis de la salud pública y el movimiento de la salud colectiva en Latinoamérica. Cuad. méd. soc. (Ros.). 1999;(75):5-30.

8. Valdivia BI. La teoría social del interaccionismo simbólico. Universidad Veracruzana. Sistema de enseñanza abierta. Carrera: Sociología/Región Orizaba. [Internet]. 2012 [citada marzo de 2012] Disponible En: http:// www.uv.mx/personal/bvaldivia/files/2012/05/Interaccionismo-PRINCIPIOS-b-.pdf.

9. Breilh J. Epidemiología Crítica: Ciencia emancipadora e interculturalidad. Buenos Aires: Lugar Editorial, 2003

10. Casallas AL. La medicina social-salud colectiva latinoamericanas: una visión integradora frente a la salud pública tradicional. Rev Cienc Salud. [Internet] 2017 [citada 28 marzo de 2020]; 15(3). Disponible en https://revistas.urosario.edu.co/xml/562/56253119009/html/index.html

11. Congreso de Colombia. Ley 1804 de 2 de agosto de 2016. Por "Por la cual se establece la política de Estado para el desarrollo integral de la primera infancia de Cero a Siempre y se dictan otras disposiciones".

12. Arrivillaga M, Ross M, Useche B, Alzate ML, Correa D. Social position, gender role, and treatment adherence among Colombian women living with HIV/AIDS: social determinants of health approach. Rev Panam Salud Publica. 2009;26(6):502-10

13. Ministerio de Salud. IV Estudio Nacional de Salud Bucal-ENSAB IV-. Situación en Salud Bucal. Bogotá. [Internet]. 2014 [Consultado 3 abril 2020]. Disponible en: https://www.minsalud.gov.co/sites/rid/Lists/BibliotecaDigital/RIDE/VS/PP/ENSAB-IV-Situacion-Bucal-Actual.pdf

14. Barón G, García PG. Representaciones sociales del proceso salud enfermedad en pacientes odontológicos del sector público y privado. Ibn Sina. Revista electrónica. [Internet]. 2012. [Citado el 3 de enero de 2012] Disponible en http://mcs.reduaz.mx/ ibnsina/2012v3t1/i_03_01-2012_1.pdf.

15. Rengifo HA. Creencias acerca de la salud oral en gestantes en tres ciudades colombianas. Rev Fac Odontol Univ Antioq 2009;20:171-178.

16. Escobar G. Sosa C. Burgos LM. Representaciones sociales del proceso de salud enfermedad bucal en madres gestantes de Medellín, Colombia. Salud Pública Mex. 2010;52:46-5.

17. Miraschi C, Ríos M, Urzúa JP, Barahona P. Calidad de vida y condición de salud oral en embarazadas chilenas e inmigrantes peruanas. Rev Perú Med Exp Salud Pública. 2009;26:455-461.

18. Saddki N, Yusoff A, Hwang YL. Factors associated with dental visit and barriers to utilization of oral health care service in a sample of antenatal mothers in Hospital Universiti Sains Malaysia. BMC Public Health 2010;10:75.
19. Amaya J, Borrero C, Ucros S. Estudio analítico del resultado del embarazo en adolescentes y mujeres de 20 a 29 ańos en Bogotá. Rev Colomb Obstet Ginecol.2005;56(3):216-224.

20. Corchuelo-Ojeda J, Gónzalez Pérez GJ. Determinantes socioeconómicos de la atención odontológica durante la gestación en Cali, Colombia. Cad Saúde Pública. 2014;30(10):2209-2218.

21. Méndez Báez MG, Pérez Bejarano NM. Características del estado periodontal en gestantes del Hospital Materno Infantil San Pablo de Asunción, Paraguay. Odontol Sanmarquina. 2018;21(3):165-172.

22. Concha Sánchez SC. Determinación social de la atención odontológica de las mujeres embarazadas de tres localidades de Bogotá [Tesis doctoral] [Bogotá]. Escuela de Medicina, Doctorado en Salud pública, Universidad Nacional de Colombia [Tesis presentada como requisito parcial para optar al título de Doctora en Salud Pública]: Bogotá; 2015. 360 p.

23. Lamarca GA, Leal MdoC, Leao ATT. Sheiham A, Vetore MV. The different roles of neighborhood and individual social capital on oral health-related quality of life during pregnancy and postpartum: a multilevel analysis. Community Dent Oral Epidemiol. 2014;47:139-150.

24. Gwengu P. Determinants or utilization of oral health services by postnatal mothers in Winterveldt, Gauteng Province, South Africa. J Oral Hyg Health. 2017;5(1):8p. DOI: $10.4172 / 2332-0702.1000222$

25. Rodríguez-Páez FG, Jiménez-Barbosa WG, JiménezGonzález CA, Coral-Córdoba AE, Ramírez-Solano PC, Ramos-Navas NR. Efecto de las barreras de acceso sobre la asistencia a citas de programa de control prenatal y desenlaces perinatales. Rev. Gerenc Polít Salud. 2014;13(27):212-227.

26. Franco-Cortes AM, Ramírez-Puerta S, Escobar-Paucar G, Isaac-Millán M, Londoño-Marín PA. Barreras de acceso a los servicios odontológicos de niños y niñas menores de 6 ańos pertenecientes a familias desplazadas. Rev CES Odont. 2010;23(2):41-48.

27. Yllesca-Yllesca I, Manrique-Chávez JE, Chávez-Reátegui BC. Características epidemiológicas de la enfermedad periodontal e higiene oral en las mujeres en etapa de embarazo y lactancia. Rev Estomatol Herediana. 2015;25(4):255-61.

28. Cartes-Velásquez R, Mardones S, Paredes C. Conocimientos y estado de salud bucal en madres beneficiarias del sistema Chile crece contigo. Rev Chil Salud Pública. 2009;13(3):136-142. 
